\title{
Efficacy of Thymol and Eugenol Against Polymicrobial Biofilm
}

\author{
Hasyrul Hamzah', Sylvia Utami Tunjung Pratiwi ${ }^{2,3}$, Triana Hertiani'2,3*
}

\begin{abstract}
1.Faculty of Pharmacy, Universitas Gadjah Mada, Yogyakarta, 55281 Indonesia 2.Department of Pharmaceutical Biology, Faculty of Pharmacy, Universitas Gadjah Mada, Yogyakarta, 55281 Indonesia ${ }^{3}$ Center for Natural Antiinfective Research (CNAIR), Faculty of Pharmacy, Universitas Gadjah Mada, Yogyakarta, Indonesia
\end{abstract}

Submitted: 10-10-2018

Revised: 20-11-2018

Accepted: 25-11-2018

*Corresponding author

Triana Hertiani

Email:

hertiani@ugm.ac.id

\begin{abstract}
Biofilms associated with human infection have high levels of pathogenicity due to their resistance to antibiotics. The discovery of an active antibiofilm agent against polymicrobial biofilms is a necessary consequence for coping with biofilmrelated infections. Thymol and Eugenol are essential oils that have potential as antibacterial and antifungal. This study aimed to determine the effectiveness of thymol and eugenol inhibits $C$. albicans, P. Aeruginosa, E. coli S. aureus and polymicrobial biofilm. Biofilm formation inhibition assay and biofilm degradation assay of thymol and eugenol were determined using microtiter broth method.The antibiofilm efficacy of thymol and eugenol towards polymicrobial biofilms were analyzed by calculating minimum biofilm inhibitor concentration (MBIC 50 ) and minimum biofilm eradication concentration $\left(\mathrm{MBEC}_{50}\right.$ ) values. The data were analyzed using Statistical Package for the Social Sciences (SPSS) with 95\% confidence level. Thymol and eugenol showed inhibitory activity against the formation of mono and polymicrobial biofilms of the microbial tested. The result also demonstrated an evidence of activity of thymol and eugenol in breaking down mono and polymicrobial biofilm. Therefore, thymol and eugenol serves as a potential source for new antibiofilm drugs towards polymicrobial biofilm.
\end{abstract}

Key words: Thymol, Eugenol, polymicrobial biofilm, MBIC $50, M_{5 B C}$

\section{INTRODUCTION}

Biofilm-related infections are an increasing health problem worldwide, especially patients suffering from immune system disorders such as HIV, cancer, organ transplants and malnutrition. Biofilm associated with human infection may contribute tomicrobial resistance towards antibiotic used. It is estimated that $65 \%$ of all hospital-related infections is caused by biofilms which includes its attachment on medical devices such as catheters and biomaterials (Furukawa et al., 2006). Biofilm in nature consists of mixture of various strain and even various species of microbes living together. The microbial diversity in polymicrobial biofilms adds complexity in the eradication process (Liu et al., 2000).

Many recent research on biofilm eradication have been done on single species microbial biofilms. However, polymicrobial biofilms can cause chronic infections while synergistic interactions in of the polymicrobials affects the physiological function of biofilms by resulting increased resistance and virulence (Burmølleet al., 2014). The formation of biofilms from both bacterial polymicrobials (Gram positive and Gram negative) as well as fungi especially from the genus $C$. albicans is responsible for the onset of disease in humans. Some literature explain that bacteria synergistically can form biofilms with other bacterial species, and physically and physiologically the structure of the biofilm becomes thicker and stronger (Anderssonet al., 2008; Cowan et al., 2003; Leriche et al., 2003).

Thymol and Eugenolare both found in plant essential oils. Thymol has a broad spectrum antimicrobial activity, and has been the subject of several studies in vitro (Dorman and Deans, 2000). Thymol and eugenol had activity inhibit mono-species biofilm $S$. aureus and E. coli (Nestro et al., 2007; Kim et al., 2016). Despite being potential as antibiofilm against single species microbes, no reports on their effects eugenol against polymicrobial biofilms consists of $P$. aeruginosa, E. coli, S. aureus, $C$. 
albicans. Research on such topic is essential to evaluate the effectiveness of eugenol and thymol, as antibiofilm in polymicrobial cultures.

\section{MATERIALS AND METHODS}

Materials used were thymol (SigmaAldrich, Germany), eugenol (Sigma-Aldrich, Germany), crystal violet (Merck, Germany), ethyl acetate (Merck, Germany), Brain Heart Infusion (Oxoid) (Merck, Germany), ethanol 95\% (Merck, Germany), nystatin (SigmaAldrich, Germany), chloramphenicol (SigmaAldrich, Germany).

\section{Equipments}

Some equipments used in this research were Laminar Air Flow, incubator (IF-2B) (Sakura, Japan), micropipette pipetman (Gilson, France), multichannel micropipette (Socorex, Swiss), microplate flat-bottom polystyrene 96 well (Iwaki, Japan), mikrotiter plate reader (Optic Ivymen System 2100-C, Spain), spectrophotometry (Genesys 10 UV Scanning, 335903) (Thermo Scientific Spectronic, USA), autoclave (Sakura, Japan), analytical scales (AB204-5, Switzerland).

\section{Bacterial Strains}

A standardstrain of Saureus ATCC 25923, Ecoli ATCC 25922, Paeruginosa ATCC 27853, wascultured in tryptic soy broth (TSB) medium and incubated at $37^{\circ} \mathrm{C}$ for $72 \mathrm{~h}$. Calbicans ATCC 10231 was cultured in Sabouraud Dextrose Broth (SDB) medium and incubated at $37^{\circ} \mathrm{C}$ withagitation $(120 \mathrm{rpm})$ for $24 \mathrm{~h}$. The optical densities $\left(\mathrm{OD}_{600}\right)$ of microbial cultures will be adjusted to 0.1 (equal of the 0.5 McFarland standard $\left.\sim 1.5 \times 10^{8} \mathrm{CFU} / \mathrm{mL}\right)$, and subsequently diluted in fresh medium to $\mathrm{OD}_{600} 0.01$ for each microbial species.

\section{Biofilm Formation Inhibition and Biofilm Eradication Assay in Vitro}

In order to determine the isolate eugenol and thymol activity toward formation and degeneration of biofilm, a microtiter plate polystyrene flat bottom 96-well was used (Pierce et al., 2008). A $100 \mu \mathrm{L}$ of C. albicans suspension $\left(10^{7} \mathrm{CFU} / \mathrm{mL}\right)$ was inserted in each well microtiter plate, $C$. albicans were incubated at $37^{\circ} \mathrm{C}$ for $90 \mathrm{~min}$ for biofilm-adhesion phase.
After the incubation, plates were washed using $150 \mu \mathrm{L}$ of sterile aquadest for three times in order to nullify the non-adhesive cells. A $100 \mu \mathrm{L}$ of media contained eugenol and thymol with different concentrations $(1 \% \mathrm{v} / \mathrm{v}, 0.5 \% \mathrm{v} / \mathrm{v}$, $0.25 \% \mathrm{v} / \mathrm{v}, 0,125 \% \mathrm{v} / \mathrm{v})$, was added to each washed-well. Media with methanol were used as diluting controland microbe suspensions were used as negative control. As the positive control, a microbe suspension with nystatin $1 \%$ was used, and a media without microbe growth was used as media control. The plates were incubated in $37^{\circ} \mathrm{C}$ for $24 \mathrm{~h}$ to form the midphase biofilm and $48 \mathrm{~h}$ for maturating phase. Plates were washed with distilled water for three times. A $125 \mu \mathrm{L}$ solution of crystal violet $1 \%$ was added to each well in order to color the formed biofilm. The plates were incubated in a room temperature for $15 \mathrm{~min}$. After incubation, the microplates were washed with running water for three times to clean the crytal violet's remains and A $200 \mu \mathrm{L}$ of ethanol $96 \%$ was added to each well. The Optical Density (OD) reading was performed with microplate reader on the wavelength of $595 \mathrm{~nm}$. The testing was performed in three times replication. The percentage of inhibition and degeneration for every eugenol and thymol concentration was counted using the formula below:

\section{$\frac{\text { Od growth control }- \text { Od sample }}{\text { Od growth control }} \times 100$}

The amount of sample that could inhibit at least $50 \%$ of the biofilm formation can be considered as minimum biofilm inhibitory concentration $\left(\mathrm{MBIC}_{50}\right)$ and minimum biofilm eradication concentration $\left(\mathrm{MBEC}_{50}\right)$ (Pratiwi et al 2015). The testing for polymicrobial biofilm C. albicans, E. coli, $P$. aeruginosa and $S$. aureus was performed under the same methods with the monospecies $C$. albicans. In other hand, the biofilm testing towards E. coli, P. aeruginosa and $S$. aureus was similar to the monospecies testing without incubation of C. albicans at $\pm 37^{\circ} \mathrm{C}$ for $90 \mathrm{~min}$ for adhesion phase. A microbial suspension that already given an antibiotic namely chloramphenicol $1 \%$ $\mathrm{v} / \mathrm{v}$, was used as control-antibiotic. 

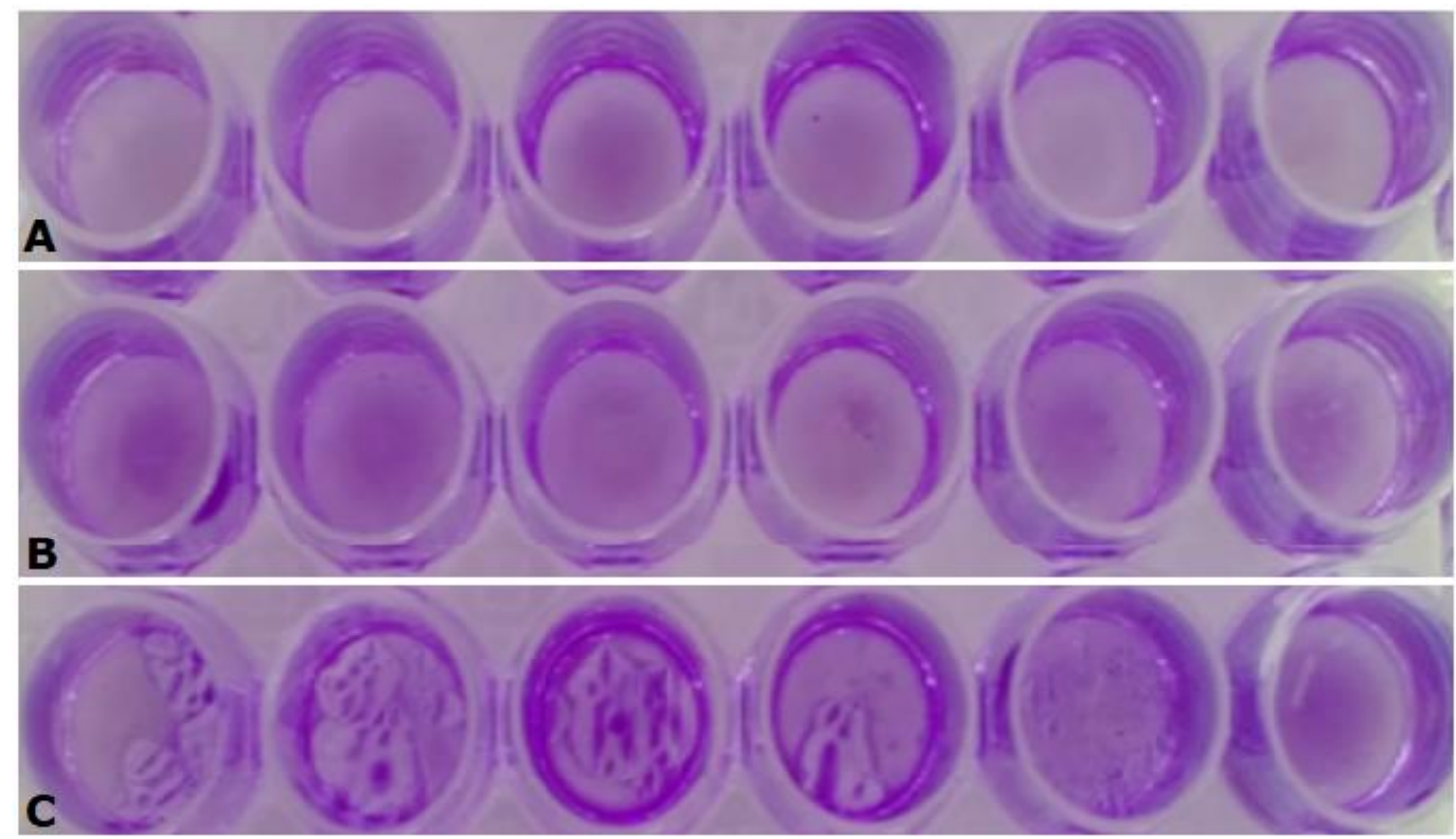

Figure 1. Crystal violet assay to see the formation of biofilm, a. Polymicrobial biofilm by adminitration of eugenol compounds; b. Polymicrobial biofilm by adminitration of thymol compounds; c. Polymicrobial biofilm without adminitration of test compound

\section{RESULT AND DISCUSSION \\ Crystal violet assay for staining biofilm}

Crystal violet staining is commonly used for quantification of biomass biofilm formation in various types of microorganisms. Crystal violet binds the negative charge on the surface of the molecule and polysaccharide to EPS so that crystal violet remains attached to the biofilm after being washed with distilled water (Peeters et al., 2008). Crystal violet was used to show the quantity of biofilm mass (Stepanovic et al., 2000). Crystal violet is bound to molecular surface negative ions and polysaccharides present in the extracellular matrix (Li et al., 2003).

Biofilm formation can be seen in the microplate base after administration of crystal violet (Figure 1). Figure 1.C shows that the intensity of crystal violet staining is very clear, this showed that the composition of biofilm numbers located in these wells is very thick and strong where the biofilm is not well formed.

Whereas in figure 1.B the intensity of crystal violet staining is not as close as in Figure $1 \mathrm{c}$. This indicates that biofilm formation is not completely formed. This inhibiting mechanism is caused by thymol compounds that inhibit polymicrobial biofim. Therefore, one bacterium was unable to form a community with other bacteria.

In figure 1. A, the intensity of staining of violet crystals is very low indicating that the biofilms formed are less than in figure 8.C and 8.B. This showed the inhibition of biofilm formation because the eugenol compound can inhibit the formation of $S$. aureus, $P$. aeruginosa, E. coli and C. albicans to form complex biofilm communities.

\section{Effectof thymol and eugenol against mono-speciesmid - phase biofilm (24h) \\ In this study we evaluated the} antibiofilm potencies of thymol and eugenol, each against polymicrobial and mono-species biofilms. We observed a decline in the rate of biofilm formation along with the increased concentrations of thymol and eugenol. The formation of $C$. albicans biofilm begins with the attachment of cells to host cells which last between $0-2 \mathrm{~h}$ continuing with hyphae formation (4-6h). The hyphae yarns form a monolayer (6-8h) that will proliferate $(8-24 \mathrm{~h})$ to then maturate (24-48h) (Ramage et al., 2001). 
Table I. Effect of thymoland eugenol against mono-species mid - phase biofilm (24h)

\begin{tabular}{cccc}
\hline Compound & Bacterial Strains & \% Inhibition & MBIC $_{50} \mathbf{v} / \mathbf{v}$ \\
\hline & P. aeruginosa & $84.10 \pm 1.26^{*}$ & $0.13 \%$ \\
Thymol 1\% & E. coli & $85.30 \pm 0.52^{*}$ & $0.29 \%$ \\
& C. albicans & $84.40 \pm 1.85^{*}$ & $0.20 \%$ \\
& S. aureus & $59.70 \pm 0.90^{*}$ & $0.51 \%$ \\
& P. aeruginosa & $86.86 \pm 1.15^{*}$ & $0.51 \%$ \\
Eugenol 1\% & E. coli & $82.87 \pm 0.62^{*}$ & $0.054 \%$ \\
& C. albicans & $81.73 \pm 1.45^{*}$ & $0.06 \%$ \\
& S. aureus & $57.56 \pm 0.51^{*}$ & $0.206 \%$ \\
Chloramphenicol 1\% & P. aeruginosa & $88.76 \pm 0.73^{*}$ & \\
& E. coli & $77.95 \pm 0.83^{*}$ & \\
Nystatin 1\% & S. aureus & $59.02 \pm 1.10^{*}$ & \\
\hline
\end{tabular}

Table II. Effect of thymol and eugenol against mid - phase polymicrobial biofilm (24h)

\begin{tabular}{ccc}
\hline Compound & \% Inhibition & MBIC $_{50} \mathbf{v} / \mathbf{v}$ \\
\hline Thymol 1\% & $84.05 \pm 0.25^{*}$ & $0.10 \%$ \\
Eugenol 1\% & $89.20 \pm 0.25^{*}$ & $0.31 \%$ \\
Chloramphenicol 1\% & $69.82 \pm 1.96^{*}$ & \\
Nystatin 1\% & $55.70 \pm 0.72^{*}$ & \\
\hline
\end{tabular}

The results of this study showed that the formation of mid-phase biofilms of $P$. aeruginosa, E. coli, C. albicans and S. aureus decreased with increasing concentrations of thymol compounds (Table I and Figure 2). Eugenol showed inhibitory activity against mid-phase mono-species and polymicrobials biofilm. The formation of mid-phase biofilms of $P$. aeruginosa, E. coli, $C$. albicans and $S$. aureus decreased with increasing concentrations of eugenol compounds (Table I and Figure. 3).

\section{Effect of Thymol and Eugenol against mid - phase polymicrobial biofilm (24h)}

The biofilm tested consisted of Grampositive bacteria i.e Staphylococus aureus and Gram-negative bacteria i.e Pseudomonas aeruginosa - Escherichia coli and yeast Candida albicans. Previous study found that bacteria synergistically can form biofilms with other bacterial species that difficult to penetrate by antibiotics compared to planktonic cells (Andersson et al., 2008). EPS matrices in biofilms may help cells survive longer by providing protection layers against antibiotics (Anderson et al., 2008; Leriche et al., 2003).

Thymol 1\% and eugenol 1\%, each showed higher activity against Nystatin and Chloramphenicol of the same concentration. It was observed a concentration dependent antibiofilm activity towards a mid-phase polymicrobial biofilm (Table II and Figure 4). This value showed that the power of inhibiting activity of thymol and eugenol compound was strong and had potential as anti-biofilm medication's candidate.

\section{Effect of Thymol and Eugenol against mono-speciesbiofilm maturation phase (48h)}

In the maturation phase, antimicrobial agents will have more difficulties to penetrate the biofilm and eugenol compounds have the potential to be antibiofilm. We observed decreasing in biofilm growth with increasing concentration of thymol and eugenol compounds, as described in (Table III, Figure 5 and 6). 
Table III. Effect of thymol and eugenol against mono-species biofilm maturation phase (48h).

\begin{tabular}{cccc}
\hline Compound & Bacterial Strains & \% Inhibition & MBIC $_{50} \mathbf{v} / \mathbf{v}$ \\
\hline & P. aeruginosa & $84.82 \pm 0.30^{*}$ & $0.22 \%$ \\
Thymol 1\% & E. coli & $80.34 \pm 0.78^{*}$ & $0.45 \%$ \\
& C. albicans & $72.63 \pm 1.41^{*}$ & $0.43 \%$ \\
& S. aureus & $55.21 \pm 1.70^{*}$ & $0.52 \%$ \\
& P. aeruginosa & $84.36 \pm 1.05^{*}$ & $0.26 \%$ \\
Eugenol 1\% & E. coli & $74.50 \pm 0.60^{*}$ & $0.51 \%$ \\
& C. albicans & $74.91 \pm 0.55^{*}$ & $0.51 \%$ \\
& S. aureus & $56.31 \pm 0.41^{*}$ & $0.62 \%$ \\
Chloramphenicol 1\% & P. aeruginosa & $79.66 \pm 0.20^{*}$ & \\
& E. coli & $81.84 \pm 0.56^{*}$ & \\
Nystatin 1\% & S. aureus & $65.31 \pm 0.78^{*}$ & \\
\hline
\end{tabular}

Table IV. Effect of thymol and eugenol against polimicrobial biofilm phase (48h).

\begin{tabular}{ccc}
\hline Compound & \% Inhibition & MBIC $_{50} \mathbf{v} / \mathbf{v}$ \\
\hline Thymol 1\% & $82.27 \pm 0.36^{*}$ & $0.26 \%$ \\
Eugenol 1\% & $71.66 \pm 0.58^{*}$ & $0.50 \%$ \\
Chloramphenicol 1\% & $74.72 \pm 0.26^{*}$ & \\
Nystatin 1\% & $50.98 \pm 3.71 *$ & \\
\hline
\end{tabular}

Thymol caused $50 \%$ damage in maturation phase of the mono-species P. aeruginosa, E. Coli, C. albicans and S. aureus. Antibiotic (chloramphenicol) and antifungal (nystatin 1\%) that used as control showed a lower biofilm inhibition compared to thymol and eugenol compound. In biofilm inhibition of mono-species P.aeruginosa and C. albicans, thymol $1 \%$ showed higher activity against Chlorampenicol and nystatin of the same concentration (Table III).

In this phase, the microbes that formed biofilm were already adhesive to the substrate, so those thymol and eugenol compounds were harder to kill the biofilm compared to the midphase. In this phase, microbes were forming a strong biofilm defense system, and built a communication cell mechanism called quorum sensing. These made the antibiotics were hard to kill and damage the bacteria that formed biofilm.

\section{Effect of Thymol and Eugenol against polimicrobial biofilm phase $(\mathbf{4 8 h})$}

Our results in figure 7 showed that thymol and eugenol with $0.5 \%$ concentration can damage the polymicrobial biofilm defense system for $50 \%$. On the concentration of $1 \%$ thymol and eugenol had inhibiting activites for $82.27 \pm 0.36 \%$ and $71.66 \pm 0.58 \%$, while in antibiotic (chloramphenicol) and antifungal (nystatin 1\%) showed a smaller inhibiting activity (Table IV). We reported that the eugenol activity on maturation phase of the polymicrobial biofilm was better compared to chloramphenicol. This showed that eugenol is potential to be developed and improved as the candidate for anti-biofilm. Eugenol at sub-inhibitory concentrations inhibited the produ-ction of virulence factors, including violacein, elastase, pyocyanin, and biofilm formation (Zhou et al., 2013)

$\mathrm{MBIC}_{50}$ values for polymicrobial biofilm maturation phase were $0.26 \% \mathrm{v} / \mathrm{v}$ (Thymol) and $0.50 \% \mathrm{v} / \mathrm{v}$ (Eugenol). This value showed that thymol and eugenol compounds had strong inhibiting activity, but eugenol $\mathrm{MBIC}_{50}$ value on the middle-phase was better compared to the maturation phase, because in the maturition phase, the heterogeneous pattern was found in the biofilm, that interacted one and another and had more complex structure. 

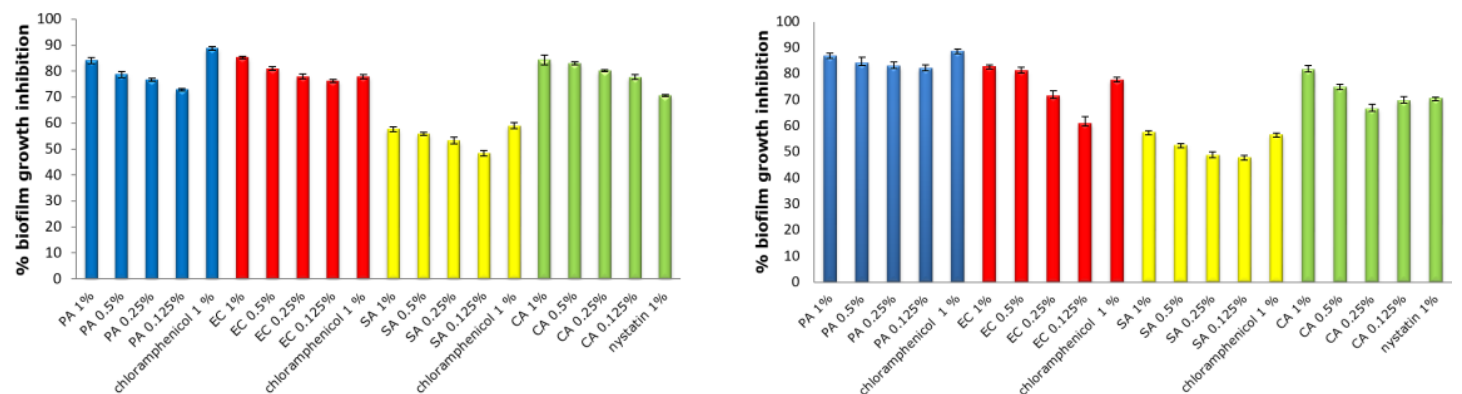

Figure 2. Effect of thymol against mono- Figure 3. Effect of eugenol against monospeciesmid - phasebiofilm. Blue $=P$. aeruginosa, speciesmid - phase biofilm. Blue $=$ P. aeruginosa, Red $=$ E.coli, Yellow $=$ C.albicans, Green $=S$. aureus $\operatorname{Red}=$ E.coli, Yellow $=$ C.albicans, Green $=S$. aureus .
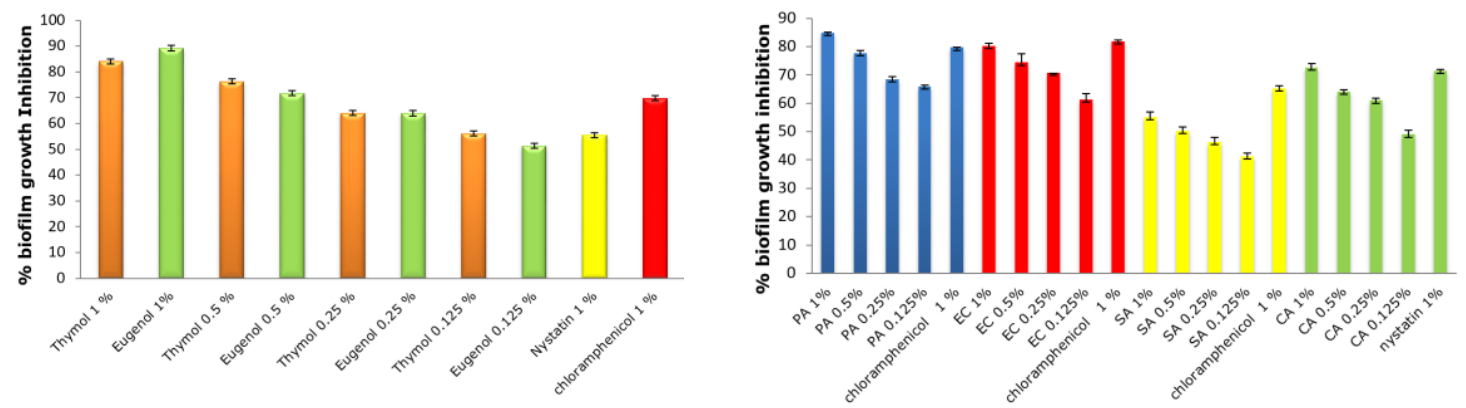

Figure 4. Effect of thymol and eugenol against Figure 5. Effect of Thymol against mono-species polymicrobial mid - phasebiofilm. Brown $=$ maturation biofilm. Blue $=P$. aeruginosa, $\operatorname{Red}=E$. Thymol, Green $=$ Eugenol, Yellow $=$ Nystatin, Red $=$ coli, Yellow $=C$. albicans, Green $=$ S. aureus . Choloramfenicol.
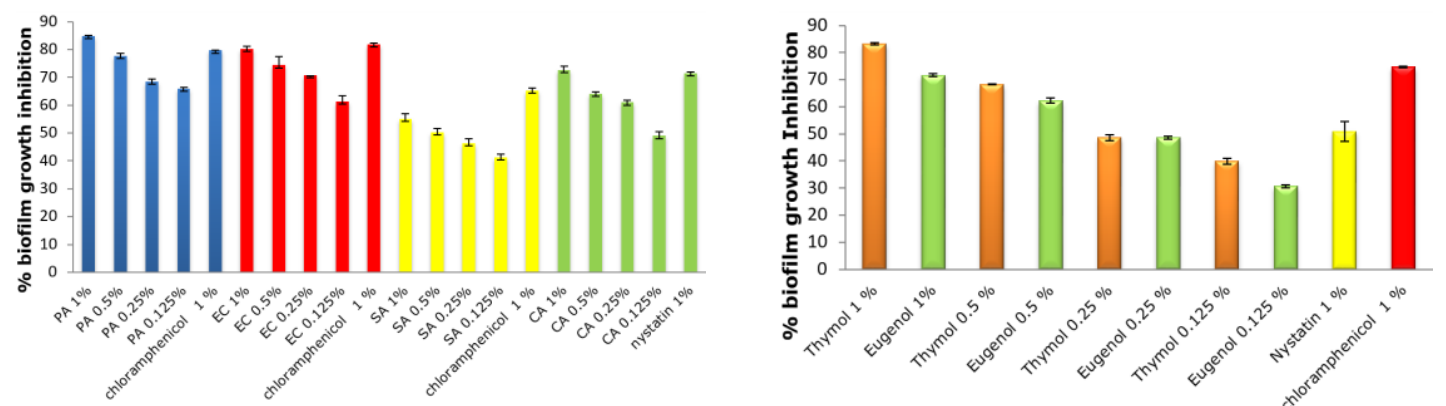

Figure 6. Effect of eugenol against mono-species Figure 7. Effect of thymol and eugenol against maturation biofilm. Blue $=P$. aeruginosa, Red $=E$. polymicrobial maturation biofilm. Brown = coli, Yellow $=C$. albicans, Green $=S$. aureus.

Thymol, Green $=$ Eugenol, Yellow $=$ Nystatin, Red $=$ Choloramfenicol.

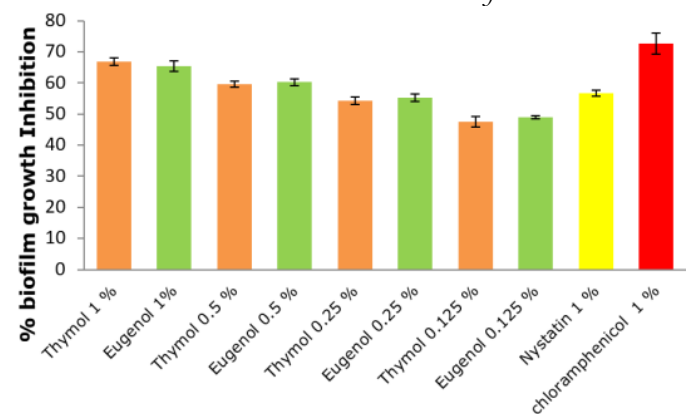

Figure 8. Effect of thymol and eugenol against polymicrobial eradication biofilm. Brown $=$ Thymol, Green $=$ Eugenol, Yellow $=$ Nystatin, Red $=$ Choloramfenicol Green 
This is in accordance with Hamzah (2017) statement that interactions in biofilm bacteria can improve the ability of other bacterial species to survive and grow in aquatic environments that trigger rapid responses and result in complex biofilm formation.

\section{Effect of Thymol and Eugenol against polimicrobial biofilm phase eradication}

In polymicrobial biofilm $P$. aeruginosa rapidly triggered $E$. coli growth, making it possible to form a boarder biofilm area. The interaction with biofilm bacteria such as $P$. aeruginosa increased the ability of E. coli to survive and grow in an aquatic environment. In polymicrobial biofilm, they were well-organized in order to ful fill each others' nutrition. We reported that thymol and eugenol degraded $50 \%$ formation of C. albicans, $P$. aeruginosa, E. coli and $S$. aureus biofilm (Figure 8).

It was harder to damage the microbes that formed biofilm compared to microbes that formed biofilm in a middle and inhibition phase. This caused by the longer growth time of biofilm, lead toformation of strong bacteria community so that antibiotics are difficult to penetrate. Matrices extracellular polymer subtances (EPS) in biofilms may help cells survive longer than if a set of conditions because EPS bound with water in an abundance amount so the microbes form a strong biofilm that had resistance toward drought, nutrients' deficiency, anti-microbial substance and other conditions that brought disadvantages to the microbes' growth. The EPS matrix on biofilms can help microorganism cells last longer than when they are in planktonic conditions, so it can help to microcortium various species of microorganisms in the degradation process (Donlan, 2002).

In this study, we reported that thymol and eugenol compound had activity of biofilm damaging. The $\mathrm{MBEC}_{50}$ value of thymol and eugenol are 0.17 and $0.24 \quad(* \mathrm{P}<0.05)$ respectively. According to this study, it can be reported that the inhibition activities of thymol and eugenol compound in biofilm degradation phase were more potent compared to the control such as nystatin 56.66 \% $\pm 0.96 \%$; while in chloramphenicol, the inhibition activitiesof thymol and eugenol compound in biofilm degradation phase were found almost similar $72.63 \pm 3.33 \%$.

Therefore effectiveness of thymol and eugenol against polymicrobial biofilm is an interesting thing to the discovery of new antibiofilm compounds as a therapeutic option for biofilm polymicrobial related infections.

\section{CONCLUSION}

Thymol and Eugenol potential as potential sources of novel antibiofilm candidates against polymicrobial biofilms $C$. albicans $-P$. aeruginosa - E. coli and S. aureus.

\section{ACKNOWLEDGEMENT}

Authors acknowledge Faculty of Pharmacy UGM for research financial support.

\section{REFERENCES}

Andersson S., Kuttuva Rajarao G., Land CJ., and Dalhammar, G., 2008. Biofilm formation and interactions of bacterial strains found in wastewater treatment systems: Biofilm formation and interactions of bacterial strains. FEMS Microbiology Letters, 283: 83-90.

Burmølle M., Ren D., Bjarnsholt T., and Sørensen, S.J., 2014. Interactions in multispecies biofilms: do they actually matter?. Trends in Microbiology. 22: 84-91.

Cowan SE., Gilbert E., Liepmann D., and Keasling, JD., 2000. 'Commensal Interactions in a Dual-Species Biofilm Exposed to Mixed Organic Compounds, Applied and Enviromental Microbiology. 66: 4481-4485.

Donlan RM., 2002. Biofilm: Microbial Life on Surfaces. Emerging Infectious Diseases 8: 881890.

Dorman HJD., Deans S. G., 2000. Antimicrobial agents from plants: antibacterial activity of plant volatile oils. $J$ Appl Microbiol. 88: 308-316.

Furukawa WF., Bryers JD., Robbins J., 2006., Biomaterial-centered infection: microbial adhesion. Science.

Hamzah H., 2017, The Effect of C-10 Massoialactone to The Culture Multispecies of Biofilm, Thesis, Universitas Gadjah Mada, Indonesia.

Kim YG., Lee JH, Gwon G, Kim SI, Park JK, Lee J., 2016. Essential Oils and Eugenols 
Inhibit Bioflm Formation and the Virulence of Escherichia coli O157:H7. Scientific reports. $6: 363777$.

Leriche V., Briandet R., dan Carpentier B., 2003. Ecology of mixed biofilms subjected daily to a chlorinated alkaline solution: spatial distribution of bacterial species suggests a protective effect of one species to another. Environmental Microbiology. 5: 64-71.

Li X., 2003. Quantitative variation of biofilms among strains in natural populations of Candida albicans. Microbiology, 149: 353362.

Liu H., Xu L., Zeng J., 2000. Role of Corrosion Products in Biofilms in Microbiologically Induced Corrosion of Carbon Steel. British Corr J. 35: 131-135.

Nestro A., Blanco A. R., Cannatelli MA., Enea V., Flamini G., Morelli I., Sudano Roccaro SA., Alonzo V., 2004. Susceptibility of methicillin-resistant staphy lococci to oregano essential oil, carvacrol and thymol. FEMS Microbiol Lett. 230: 191195.

Peeters E., Nelis, HJ., and Coenye T., 2008. Comparison of multiple methods for quantification of microbial biofilms grown in microtiter plates. Journal of Microbiological Methods, 72: 157-165.
Pierce CG., Uppuluri P., Tristan AR., Wormley FL., Mowat E, Ramage G., 2008. A Simple and Reproducible 96 Well Plate-Based Method for The Formation of Fungal Biofilms and Its Application to Antifungal Susceptibility Testing. Nat Protoc. 3: 1494-1500.

Pratiwi SUT., Lagendijk EL., de Weert S., Hertiani T., Idroes R., Van Den Hondel CAMJJ., 2015. Effect of Cinnamomumburmannii Nees ex Bl. and Massoiaaromatica Becc. Essential oils on planktonic growth and biofilm formation of Pseudomonas aeruginosa and Staphylococcus aureus in vitro. International Journal of Applied Research in Natural Product.8: 1-13.

Ramage G., VandeWalle K., Wickes BL., Lopez-Ribot JL., 2001. Standardized Method for In Vitro Antifungal Susceptibility Testing of Candida albicans Biofilms. Antimicrobial Agents and Chemotherapy. 45: 2475-2479.

Stepanović S., Vuković D., Dakić I., Savić B., and Švabić-Vlahović M., 2000. A modified microtiter-plate test for quantification of staphylococcal biofilm formation. Journal of Microbiological Methods, 40: 175-179.

Zhou L., Zheng H., Tang Y., Yu W., Gong Q., 2013. Eugenol inhibits quorum sensing at sub-inhibitory concentrations. Biotechnol Lett. 35: 631-637. 